TRANSACTIONS OF THE

AMERICAN MATHEMATICAL SOCIETY

Volume 350, Number 5, May 1998, Pages 1977-1990

S 0002-9947(98)01932-1

\title{
HYPERBOLIC COMPLETE MINIMAL SURFACES WITH ARBITRARY TOPOLOGY
}

\author{
F. J. LÓPEZ
}

\begin{abstract}
We show a method to construct orientable minimal surfaces in $\mathbb{R}^{3}$ with arbitrary topology. This procedure gives complete examples of two different kinds: surfaces whose Gauss map omits four points of the sphere and surfaces with a bounded coordinate function. We also apply these ideas to construct stable minimal surfaces with high topology which are incomplete or complete with boundary.
\end{abstract}

\section{INTRODUCTION}

The literature on minimal surfaces is vast. Nowadays we know a wealth of orientable complete hyperbolic minimal surfaces, but these examples are somewhat rare.

Most of them have been obtained by using the Weierstrass representation for minimal surfaces and are parametrized by the unit disc $\mathbb{D}$.

However, to show examples with nontrivial topology is a quite difficult problem: we first have to specify a Riemann surface, then guess the complex theoretic data, and finally check period closing and completeness.

The period problem and the completeness are the main difficulty, and this is the reason we don't know a lot of examples.

It is only due to remarkable effort and persistence that this somewhat implicit method has given a satisfactory geometric understanding for some families of such surfaces.

In this paper we present a method which answers the above question. We obtain an analytically clear general construction for orientable complete hyperbolic minimal surfaces with arbitrary topology. As a consequence, this method yields complete nonflat examples of two different kinds: surfaces whose Gauss map omits four points of the sphere and surfaces with a bounded coordinate funtion.

R. Osserman [O], F. Xavier [X] and H. Fujimoto [FU1, FU2] studied the size of the spherical image of an orientable complete nonflat minimal surface. The last author obtained the best possible theorem, and proved that the number of exceptional values of the Gauss map is at most four. Indeed, there are many kinds of complete minimal surfaces whose Gauss map omits four points of the sphere. Among these examples we emphasize Sherk's classical doubly periodic surface and

Received by the editors May 20, 1996 and, in revised form, August 12, 1996.

1991 Mathematics Subject Classification. Primary 53A10; Secondary 53C42.

Key words and phrases. Minimal surfaces. Riemann surfaces.

Research partially supported by DGICYT Grant No. PB94-0796.

(C)1998 American Mathematical Society 
those described by K. Voss in [V] (see also [O]). Voss's examples are simplyconnected and, of course, of hyperbolic type.

In this paper we prove

Theorem A. For any $n \in \mathbb{N}, n \geq 1$, there exist a family of orientable complete nonflat minimal surfaces of genus $n$ and finite topology whose Gauss maps omit four points of the sphere.

Concerning orientable complete nonflat examples with a bounded coordinate function, the first existence result was obtained by L.P. Jorge and F. Xavier [JX1]. Later F.F. Brito [B1] showed a new method to construct the same kind of surfaces. Many other authors, including H. Rosenberg and E. Toubiana [RT], F.J. López [L], C. Costa and P.A.Q. Simoes [CS] and F. Brito [B2], have obtained complete examples with nontrivial topology contained in a slab of $\mathbb{R}^{3}$. Their methods are based on those described by Jorge-Xavier and Brito.

We use both Jorge-Xavier's and Brito's constructions in a different way to obtain

Theorem B. For any $n \in \mathbb{N}, n \geq 1$, there exist a family of orientable complete nonflat minimal surfaces of genus $n$ having finite topology and contained in a slab of $\mathbb{R}^{3}$.

Finally, we mention that our construction also yields stable examples with arbitrary topology which are incomplete or complete with boundary. We emphasize the following result:

Theorem C. For any $n \in \mathbb{N}, n \geq 1$, there exist a family of complete nonflat stable minimal surfaces of genus $n$ with finite topology and nonempty boundary.

In Theorem $\mathrm{C}$, of course we can replace completeness by compactness.

We can find families of surfaces satisfying Theorem A, B or C with exactly three ends, and it is natural to deduce from our method the existence of examples with any number of ends greater than or equal to three.

Furthermore, any surface in the above three families can be deformed, by surfaces of the same type and preserving the topology and number of ends along the deformation, into a branched covering of a plane.

For the details, see section 3 .

\section{Preliminaries on Minimal Surfaces and Riemann Surfaces}

Thoroughout this section, $M$ will be a Riemann surface with piecewise analytic boundary $\partial M$. This means that $M$ can be considered as a subset of an open Riemann surface $M^{\prime}$, the conformal structure of $M-\{\partial M\}$ is that induced by $M^{\prime}$, and $\partial M$ consists of a set of piecewise analytic curves in $M^{\prime}$, each of them homeomorphic to either $\mathbb{R}$ or $\mathbb{S}^{1}$. The case $\partial M=\emptyset$ is allowed.

Functions and 1-forms meromorphic (holomorphic) on $M$ are by definition the restriction of functions and 1-forms meromorphic (holomorphic) on $M^{\prime}$.

Let $\Phi_{k}, k=1,2,3$, denote three holomorphic 1-forms on $M$ satisfying

$$
\left(\sum_{k=1}^{3} \Phi_{k}{ }^{2}\right)(P)=0, \quad\left(\sum_{k=1}^{3}\left|\Phi_{k}\right|^{2}\right)(P) \neq 0
$$

for any $P \in M$, and

$$
\operatorname{Re}\left(\int_{\alpha} \Phi_{k}\right)=0, \quad k=1,2,3,
$$

for any closed curve $\alpha \subset M$. 
Then, given $P_{0} \in M$, the map $x: M \rightarrow \mathbb{R}^{3}$ defined by

$$
x(P)=\operatorname{Re}\left(\int_{P_{0}}^{P}\left(\Phi_{1}, \Phi_{2}, \Phi_{3}\right)\right)
$$

provides a conformal minimal inmersion of $M$ in $\mathbb{R}^{3}$. Conversely, up to translations, any conformal minimal inmersion $x$ of $M$ in $\mathbb{R}^{3}$ is obtained in this way.

Moreover, $g=\left(\Phi_{2}-i \Phi_{1}\right) /\left(i \Phi_{3}\right)$ is the Gauss map of $x$, and the 1-forms $\Phi_{k}$, $k=1,2,3$, are determined by $g$ and the 1 -form $\Phi_{3}$.

Furthermore, $d s^{2}=\sum_{k=1}^{3}\left|\Phi_{k}\right|^{2}$ is the metric induced by $x$ on $M$; that is, if $z$ is a local conformal parameter on $M$ and $\Phi_{k}(z)=f_{k}(z) d z$, then

$$
d s^{2}=\sum_{k=1}^{3}\left|f_{k}(z)\right|^{2}|d z|^{2} .
$$

By definition, $\left(M,\left(\Phi_{k}\right)_{k=1,2,3}\right)$ is the Weierstrass representation of $x$. For details see $[\mathrm{O}]$.

To finish this section, we recall some basic topics on compact Riemann surfaces. We suppose that $M$ is a compact Riemann surface (without boundary) of genus $n \geq 1$.

Take $\left\{Q_{0}, Q_{1}, \ldots, Q_{k}\right\} \subset M$, a set of $k+1$ different points, and define $M_{0}=$ $M-\left\{Q_{0}, Q_{1}, \ldots, Q_{k}\right\}$.

For the following results, see $[\mathrm{F}-\mathrm{K}]$, pp. 79-90. Let $H_{h o l}^{1}(M), H_{h o l}^{1}\left(M_{0}\right)$ denote the first holomorphic de Rham cohomology group of $M, M_{0}$ respectively. By definition, this group is the vector space of holomorphic differentials on $M, M_{0}$ respectively, factored by the subspace of exact holomorphic differentials.

Let $\left\{\xi_{1}, \ldots, \xi_{n}\right\}$ be a basis of the $\mathbb{C}$-linear vector space of holomorphic 1-forms on $M$ (remember that $\left.\operatorname{dim}_{\mathbb{C}}\left(H_{\text {hol }}^{1}(M)\right)=n\right)$. Consider the Weierstrass "gap" sequence at $Q_{0}: 1=q_{1}<\ldots<q_{n}<2 n$ (see $[\mathrm{F}-\mathrm{K}]$, p. 81$)$ and take $\xi_{n+j}$ a meromorphic 1-form on $M$ with a pole of order $q_{j}+1$ at $Q_{0}$ and holomorphic on $M-\left\{Q_{0}\right\}$, $j=1, \ldots, n$. Finally denote by $\xi_{2 n+j}$ a meromorphic 1 -form on $M$ with simple poles at both $Q_{0}, Q_{j}$ and holomorphic on $M-\left\{Q_{0}, Q_{j}\right\}, j=1, \ldots, k$.

Theorem 1 ([F-K, p. 90]). Each element of $H_{h o l}^{1}\left(M_{0}\right)$ is uniquely represented by a meromorphic differential in the linear span of the $2 n+k$ linearly independent differentials $\left\{\xi_{1}, \ldots, \xi_{2 n+k}\right\}$.

Let $f$ be a nonconstant meromorphic function on $M$ with a pole of order $m_{0}$ at $Q_{0}, m_{0}>q_{n}$, and holomorphic on $M-\left\{Q_{0}\right\}$. Suppose that the canonical divisor of $d f$ is given by

$$
[d f]=\frac{\prod_{j=1}^{k} Q_{j}^{m_{j}}}{Q_{0}^{m_{0}+1}} .
$$

The following lemma is a easy consequence of the Weierstrass "gap" theorem:

Lemma 1. There exists a meromorphic function $v$ on $M$ satisfying the following conditions:

1. $v$ is holomorphic on $M-\left\{Q_{0}, Q_{1}, \ldots, Q_{k}\right\}$.

2. The order of the pole of $v$ at $Q_{j}$ is greater than or equal to $m_{j}+2, j=1, \ldots, k$.

3. The order of the pole of $v$ at $Q_{0}$ is not a multiple of $m_{0}$. In particular, $v$ is not a rational function of $f$. 
Proof. If $1=p_{1}^{j}<\ldots<p_{n}^{j}<2 n$ is the Weierstrass "gap" sequence at $Q_{j}, j=$ $1, \ldots, k$, there exists a meromorphic function $v_{j}$ holomorphic on $M-\left\{Q_{j}\right\}$ and with a pole at $Q_{j}$ of order $s_{j}$, where $s_{j}$ is the first non- "gap" greater than or equal to $m_{j}+2$. Analogously, there exists a meromorphic function $v_{0}$ holomorphic on $M-\left\{Q_{0}\right\}$ and with a pole at $Q_{0}$ of order $s_{0}$, where $s_{0}$ is the first non- "gap" at $Q_{0}$ such that $\operatorname{gcd}\left(s_{0}, m_{0}\right)=1$. To obtain the lemma, define $v=\sum_{j=0}^{k} v_{j}$.

\section{Main Theorems}

Let $\mathbb{D}=\{z \in \mathbb{C}:|z|<1\}$ be the unit disk and let $A$ denote a set such that $\mathbb{D} \subset A \subset \overline{\mathbb{D}}$. We suppose that the boundary of $A, \partial A:=A \cap \partial \mathbb{D}$, is the union of a countable (possibly void) set of open arcs contained in $\partial \mathbb{D}$. It is clear that $\partial A=\emptyset$ if and only if $A=\mathbb{D}$ and $\partial A=\partial \mathbb{D}$ if and only if $A=\overline{\mathbb{D}}$. In a natural way $A$ is a Riemann surface with (possibly void) boundary.

Let $x: A \longrightarrow \mathbb{R}^{3}$ be a conformal nonflat minimal immersion of $A$ in $\mathbb{R}^{3}$, and denote by $g$ its Gauss map.

We are going to show a method to produce minimal immersions with arbitrary topology derived from $x$. First, we give a brief outline of the global strategy.

Let $M$ be a compact Riemann surface without boundary of genus $n \geq 1$ and take $G: M \longrightarrow \overline{\mathbb{C}}, \overline{\mathbb{C}}=\mathbb{C} \cup\{\infty\}$, a nonconstant meromorphic function. As we will see later, we can choose $G$ in such a way that the set $G^{-1}(\mathbb{D})$ has a connected component $C$ conformally equivalent to a compact surface of genus $n$ minus a finite number of pairwise disjoint disks with piecewise analytic boundary. In this case, we define $C_{A}$ as the topological closure of $C$ in $G^{-1}(A)$. Obviously $C_{A}$ is a Riemann surface with (possibly void) piecewise analytic boundary. Furthermore $\partial A=\emptyset$ implies $\partial C_{A}=\emptyset$ and $C=C_{A}$, and when $A=\overline{\mathbb{D}}$ then $C_{A}$ is a compact Riemann surface with piecewise analytic boundary.

Our main achievement is to construct conformal minimal immersions $y: C_{A} \longrightarrow$ $\mathbb{R}^{3}$ whose Gauss map is equal to $g \circ\left(G_{\mid C_{A}}\right)$. This construction is essentially based on a good choice of both the meromorphic function $G$ on $M$ and the Weierstrass data on $C_{A}$. The main difficulty is, of course, to solve the period problem.

This procedure will obviously preserve any property that depends on the Gauss map and is invariant after composing with a branched covering: for instance, the size of the image of the Gauss map and the stability properties of the surface.

Another important preserved property is the completeness.

We now explain the details.

The first step consists of fixing a point $Q_{0} \in M$ and taking a nonconstant meromorphic function $f$ on $M$ with a pole of order $m_{0}$ at $Q_{0}$ and holomorphic on $M-\left\{Q_{0}\right\}$. Denoting by $1=q_{1}<\ldots<q_{n}<2 n$ the Weierstrass "gap" sequence at $Q_{0}$, we assume that $m_{0}>q_{n}$. Write the canonical divisor of $d f$ as

$$
[d f]=\frac{\prod_{j=1}^{k} Q_{j}^{m_{j}}}{Q_{0}^{m_{0}+1}}
$$

and let $v$ be the function in Lemma 1 .

Then, define $M_{0}=M-\left\{Q_{0}, Q_{1}, \ldots, Q_{k}\right\}$ and let $\left\{\xi_{1}, \ldots, \xi_{2 n+k}\right\}$ be the basis of $H_{h o l}^{1}\left(M_{0}\right)$ given in Theorem 1.

We deal with the following family of meromorphic functions:

$$
\left\{G^{\Lambda}: \Lambda=\left(\lambda, \lambda_{1}, \ldots, \lambda_{k+2 n}\right) \in \mathbb{C}^{2 n+k+1}\right\},
$$


where

$$
G^{\Lambda}=\lambda v+\frac{\sum_{j=1}^{2 n+k} \lambda_{j} \xi_{j}}{d f} .
$$

Remark 1. For any $\Lambda \in \mathbb{C}^{2 n+k+1}$, the function $G^{\Lambda}$ is holomorphic on $M_{0}$.

Moreover, if $\Lambda \neq 0$ then $G^{\Lambda}$ is not constant. Furthermore, when $\lambda \neq 0$ the function $G^{\Lambda}$ has a pole at any point $Q_{j}, j \in\{0,1, \ldots, k\}$.

To check the second part of the remark, observe that, by Lemma 1 and the definition of the 1 -forms $\xi_{1}, \ldots, \xi_{2 n+k}$ in the preceding section, the function $v$ has a pole at $Q_{j}$ of order greater than the pole of $\sum_{j=1}^{2 n+k} \lambda_{j} \frac{\xi_{j}}{d f}$ at this point, $j=0,1, \ldots, k$. Hence, $\lambda \neq 0$ implies that $G^{\Lambda}$ has a pole at $Q_{j}, j=0,1, \ldots, k$. If $\Lambda \neq 0$ and $\lambda=0$, use that $\xi_{1}, \ldots, \xi_{2 n+k}$ are linearly independent (see Theorem 1) to get $G^{\Lambda} \neq$ constant.

We denote $|\Lambda|=|\lambda|+\sum_{j=1}^{2 n+k}\left|\lambda_{j}\right|$.

Lemma 2. There exists a constant $\varepsilon>0$ such that if $|\Lambda|<\varepsilon$, then the set $\left(G^{\Lambda}\right)^{-1}(\mathbb{D})$ has a connected component $C^{\Lambda}$ conformally equivalent to a compact surface of genus $n$ minus at most $k+1$ pairwise disjoint disks with piecewise analytic boundary. Furthermore, if $\lambda \neq 0$ then $\left(G^{\Lambda}\right)^{-1}(\mathbb{D})$ has exactly $k+1$ holes.

Proof. Remember that the function $G^{\Lambda}$ is holomorphic on $M_{0}$ and, by Remark 1, $\lambda \neq 0$ implies that $G^{\Lambda}$ has a nontrivial pole at any $Q_{j}, j=0,1, \ldots, k$.

Let $\left\{\bar{D}_{j}: j=0,1, \ldots, k\right\}$ denote set of closed pairwise disjoint conformal disks such that $Q_{j} \in D_{j}$ (here $D_{j}$ is the interior of $\bar{D}_{j}$ ), $j=0,1, \ldots, k$.

From our choice of $f$ and $\xi_{j}$, the functions $\xi_{j} / d f$ are holomorphic on $M_{0} \cup\left\{Q_{0}\right\}$, $j=1, \ldots, 2 n+k$. Hence for each $j \in\{1, \ldots, 2 n+k\}$ we can define

$$
K_{j}=\operatorname{Max}\left\{\left|\xi_{j} / d f\right|(P): P \in M-\left(\bigcup_{i=0}^{k} D_{j}\right)\right\}>0
$$

and analogously

$$
K_{0}=\operatorname{Max}\left\{|v|(P): P \in M-\left(\bigcup_{i=0}^{k} D_{j}\right)\right\}>0 .
$$

Then take $K>\operatorname{Max}\left\{K_{j}: j=0,1, \ldots, 2 n+k\right\}>0$ and $0<\varepsilon \leq \frac{1}{(2 n+k+1) K}$.

Thus, for $|\Lambda|<\varepsilon$, it is not hard to check that

$$
M-\left(\bigcup_{i=0}^{k} D_{j}\right) \subset\left(G^{\Lambda}\right)^{-1}(\mathbb{D})
$$

We are going to study the topology of $\left(G^{\Lambda}\right)^{-1}(\mathbb{D}),|\Lambda|<\varepsilon$. The case $\Lambda=0$ is trivial, and so we suppose that $\Lambda \neq 0$.

First we observe that $\left(G^{\Lambda}\right)^{-1}(\overline{\mathbb{C}}-\overline{\mathbb{D}})$ contains as many connected components as $G^{\Lambda}$ has different poles.

If $G^{\Lambda}$ has a pole at $Q_{j}, j \in\{0,1, \ldots, k\}$, then this point is the unique pole lying in $\bar{D}_{j}$. Since $\left|G^{\Lambda}\right|_{\mid \partial D_{j}}<1$, there is a connected component of $\left(G^{\Lambda}\right)^{-1}(\overline{\mathbb{C}}-\overline{\mathbb{D}})$ including $Q_{j}$ and contained in $D_{j}$. Hence each pole of $G^{\Lambda}$ is included in a different connected component of $\left(G^{\Lambda}\right)^{-1}(\overline{\mathbb{C}}-\overline{\mathbb{D}})$. If the set $\left(G^{\Lambda}\right)^{-1}(\overline{\mathbb{C}}-\overline{\mathbb{D}})$ had another connected component, call it $\Omega$, containing no pole of $G^{\Lambda}$, then the function $\left(G^{\Lambda}\right)_{\mid \Omega}$ would be holomorphic, with $\left|G^{\Lambda}\right|_{\mid \Omega}>1$ and $\left|G^{\Lambda}\right|_{\mid \partial \Omega}=1$. By the maximum principle 
for harmonic functions (applied to $\log \left(\left|G^{\Lambda}\right|\right)_{\mid \Omega}$ ), this function would be constant, a contradiction (recall that $\Lambda \neq 0$ and Remark 1 ).

Let $C^{\Lambda}$ be the connected component of $\left(G^{\Lambda}\right)^{-1}(\mathbb{D})$ containing $M-\left(\bigcup_{i=0}^{k} D_{j}\right)$.

It is now clear that $\partial C^{\Lambda} \subset \bigcup_{i=0}^{k} D_{j}$ and $\partial C^{\Lambda}$ has at least as many components as $G^{\Lambda}$ has different poles. On the other hand $\partial C^{\Lambda}$ is the nodal set of the harmonic function $\log \left(\left|G^{\Lambda}\right|\right)$, and so it is the union of a finite number of properly immersed curves which are embedded and analytic except at a discrete (indeed finite) set of points where some of the curves meet in a equiangular way (see $[\mathrm{CH}]$ for a more general setting). Note that the finite set of singular points is void if and only if $G^{\Lambda}$ has no branch points on $\partial C^{\Lambda}$.

When $Q_{j}$ is a pole of $G^{\Lambda}$, the set $\alpha_{j}=\partial C^{\Lambda} \cap D_{j}$ consists of exactly one piecewise analytic curve homeomorphic to $\mathbb{S}^{1}$. To see this, observe that $\alpha_{j}$ bounds only a compact domain in $D_{j}$. Otherwise there would be at least two compact domains with boundary contained in $\alpha_{j}$, and so one of them, call it $\Omega$, would not contain $Q_{j}$. Therefore $\Omega$ would be a connected component of $\left(G^{\Lambda}\right)^{-1}(\overline{\mathbb{C}}-\overline{\mathbb{D}})$ containing no pole of $G^{\Lambda}$, which is absurd by the maximum principle as above.

Putting together all we have obtained, we deduce that $C^{\Lambda}$ is conformally a compact surface of genus $n$ minus as many pairwise disjoint disks (bounded by the curves $\alpha_{j}$ ) as the function $G^{\Lambda}$ has poles. In particular, by Remark $1, \lambda \neq 0$ implies that $C^{\Lambda}$ has exactly $k+1$ holes.

In what follows, we assume that $|\Lambda|<\varepsilon$, where $\varepsilon$ is the constant given in Lemma 2 .

Remark 2. If we denote by $C_{A}^{\Lambda}$ the topological closure of $C^{\Lambda}$ in $\left(G^{\Lambda}\right)^{-1}(A)$, then $C_{A}^{\Lambda}$ is a Riemann surface with (possibly void) piecewise analytic boundary. Furthermore, $A=\mathbb{D}$ implies $C^{\Lambda}=C_{A}^{\Lambda}$, and $A=\overline{\mathbb{D}}$ yields that $C_{A}$ is a compact Riemann surface with piecewise analytic boundary.

For our reasoning, we can take an arbitrary compact Riemann sruface $M$ and any point $Q_{0} \in M$. Furthermore, the Riemann-Roch theorem provides an enormous family of suitable functions $f$ and $v$. It is natural to think that this generality yields, by Lemma 2, a large family of genus $n$ surfaces with an arbitrarily high finite number of holes (or ends).

However, the most interesting success should be to obtain surfaces with the lowest possible number of ends. At this point, we have:

Lemma 3. For a suitable choice of the Riemann surface $M$, the point $Q_{0} \in M$ and the functions $f$ and $v$, the surfaces $C^{\Lambda}$ have at most three holes. Moreover, if $\lambda \neq 0$ then they have exactly three holes.

Proof. Consider Riemann surface of the compact genus $n, n>0$, defined by

$$
M=\left\{(z, w) \in \overline{\mathbb{C}}^{2}: w^{2 n+1}=z^{2}-1\right\}
$$

with the canonical complex structure.

Define $Q_{0}=(\infty, \infty), Q_{1}=(1,0), Q_{2}=(-1,0)$ and $\left\{P_{1}, \ldots, P_{2 n+1}\right\}=z^{-1}(0)$. Then take $f=z$ and $v=\frac{z^{3}}{w\left(z^{2}-1\right)}$.

It is clear that the canonical divisor of $d f$ and the principal divisor of $v$ are given by

$$
[d f]=\frac{Q_{1}^{2 n} Q_{2}^{2 n}}{Q_{0}^{2 n+2}}, \quad[v]=\frac{P_{1}^{3} \cdots P_{2 n+1}^{3}}{Q_{0}^{2 n-1} Q_{1}^{2 n+2} Q_{2}^{2 n+2}} .
$$


The highest Weierstrass gap of $M$ at $Q_{0}$ is always less than $2 n$ and less than the pole order of $f$ at $Q_{0}$. Furtheromore, $v$ satisfies conditions 1, 2 and 3 in Lemma 1.

Hence, we can apply above construction, and Lemma 2 yields surfaces with at most three ends.

Let $\left\{\bar{D}_{j}: j=0,1, \ldots, k\right\}$ be the set of closed pairwise disjoint conformal disks chosen in the proof of Lemma 2. Recall that $Q_{j} \in D_{j}, j=0,1, \ldots, k$, and $\bar{D}_{j} \cap \bar{D}_{i}=$ $\emptyset, i \neq j$.

Let $\left\{\gamma_{1}, \ldots, \gamma_{2 n}\right\}$ denote a homology basis of $M$ contained in $M-\left(\bigcup_{j=0}^{k} \overline{D_{j}}\right)$, and put $\gamma_{2 n+j}=\partial D_{j}, j=1, \ldots, k$. It is clear that the set $\left\{\gamma_{1}, \ldots, \gamma_{2 n+k}\right\}$ contains a homology basis of $C_{A}^{\Lambda}$ (in fact, it is a homology basis when $C_{A}^{\Lambda}$ has $k+1$ holes).

Let $\left(A,\left(\Phi_{k}\right)_{k=1,2,3}\right)$ denote the Weierstrass representation of $x: A \longrightarrow \mathbb{R}^{3}$. Remember that the Gauss map $g$ of $x$ and the 1 -form $\Phi_{3}$ determine the Weierstrass data, and let $h$ be the holomorphic function on $A$ defined by $h(z) d z=\left(\Phi_{3} / g\right)(z)$.

For each $\Delta=\left(\delta_{1}, \ldots, \delta_{2 n+k}\right) \in \mathbb{C}^{2 n+k}$, define the meromorphic function

$$
F^{\Delta}=G^{(0, \Delta)}=\sum_{j=1}^{2 n+k} \delta_{j} \frac{\xi_{j}}{d f} .
$$

Take the following Weierstrass data on $C_{A}^{\Lambda}$ :

$$
g^{\Lambda}=g \circ G^{\Lambda}, \quad \Phi_{3}^{\Lambda, \Delta}=\operatorname{Exp}\left(F^{\Delta}\right)\left(h \circ G^{\Lambda}\right) d f .
$$

Remark 3. When $C_{A}^{\Lambda}$ has $k+1$ holes (for instance, if $\lambda \neq 0$ (see Remark 1 and Lemma 2)), the zeroes and poles of $d f$ (see (4)) are not contained in $C_{A}^{\Lambda}$. Hence, because $\operatorname{Exp}\left(F^{\Lambda}\right)$ is holomorphic and never vanishes on $M_{0}$, the three 1-forms associated to $g^{\Lambda}$ and $\Phi_{3}^{\Lambda, \Delta}$, namely

$$
2 \Phi_{1}^{\Lambda, \Delta}=\left(g^{\Lambda}-1 / g^{\Lambda}\right) \Phi_{3}^{\Lambda, \Delta}, 2 \Phi_{2}^{\Lambda, \Delta}=i\left(g^{\Lambda}+1 / g^{\Lambda}\right) \Phi_{3}^{\Lambda, \Delta}, \Phi_{3}^{\Lambda, \Delta},
$$

satisfy (1).

Therefore, fixing $P_{0} \in C_{A}^{\Lambda}$ and taking into account (3), we see that the function $x^{\Lambda, \Delta}: C_{A}^{\Lambda} \longrightarrow \mathbb{R}^{3}$ given by

$$
2 x^{\Lambda, \Delta}=\operatorname{Real} \int_{P_{0}}\left(g^{\Lambda}-1 / g^{\Lambda}, i\left(g^{\Lambda}+1 / g^{\Lambda}\right), 2\right) \Phi_{3}^{\Lambda, \Delta}
$$

defines a multivaluated conformal minimal immersion of $C_{A}^{\Lambda}$ in $\mathbb{R}^{3}$.

Moreover, $x^{\Lambda, \Delta}$ is well defined on $C_{A}^{\Lambda}$ if and only if (2) holds, that is,

$$
\operatorname{Real}\left(\int_{\gamma_{j}} \Phi_{i}^{\Lambda, \Delta}\right)=0, \quad i=1,2,3, j=1, \ldots, 2 n+k .
$$

The following theorem answers this problem:

Theorem 2. There exist an open subset $U \subset \mathbb{C}, 0 \in U$, and a differentiable (in fact real analytic and in general nonconstant) $\operatorname{map} \Psi=\left(\Psi_{1}, \Psi_{2}\right): U \longrightarrow \mathbb{C}^{2 n+k} \times \mathbb{C}^{2 n+k}$ such that $\left|\left(\lambda, \Psi_{1}(\lambda)\right)\right|<\varepsilon, \forall \lambda \in U$, and the minimal immersion $x^{\lambda}: C_{A}^{\left(\lambda, \Psi_{1}(\lambda)\right)} \longrightarrow$ $\mathbb{R}^{3}$ given by $x^{\lambda}=x^{\left(\lambda, \Psi_{1}(\lambda)\right), \Psi_{2}(\lambda)}$ is well defined (i.e., it has no real periods).

Proof. Let $\Sigma$ denote the open set

$$
\Sigma=\left\{(\Lambda, \Delta) \in \mathbb{C}^{2 n+k+1} \times \mathbb{C}^{2 n+k}:|\Lambda|<\varepsilon\right\} .
$$


We will use the notation

$$
\lambda_{j}=x_{2 j-1}+i x_{2 j}, \quad \delta_{j}=y_{2 j-1}+i y_{2 j}, \quad j=1, \ldots, 2 n+k,
$$

where $x_{i}, y_{i} \in \mathbb{R}, i=1, \ldots, 4 n+2 k$. For each $j \in\{1, \ldots, 2 n+k\}, i \in\{1,2,3\}$, define $p^{i, j}: \Sigma \longrightarrow \mathbb{R}$ as follows:

$$
p^{i, j}(\Lambda, \Delta)=\operatorname{Real}\left(\int_{\gamma_{j}} \Phi_{i}^{\Lambda, \Delta}\right) .
$$

Define also, for each $j \in\{1, \ldots, 2 n+k\}$,

$$
p^{4, j}(\Lambda, \Delta)=\operatorname{Im}\left(\int_{\gamma_{j}} \Phi_{3}^{\Lambda, \Delta}\right) .
$$

Let $P: \Sigma \longrightarrow \mathbb{R}^{4(2 n+k)}$ be the function given by

$$
P=\left(p^{i, 1}, \ldots, p^{i, 2 n+k}\right)_{i=1,2,3,4}
$$

and write, for $i \in\{1,2,3,4\}$,

$$
\begin{gathered}
A_{i}=\left(\begin{array}{cccc}
\frac{\partial p^{i, 1}}{\partial x_{1}} & \frac{\partial p^{i, 1}}{\partial x_{2}} & \cdots & \frac{\partial p^{i, 1}}{\partial x_{4 n+2 k}} \\
\frac{\partial p^{i, 2}}{\partial x_{1}} & \frac{\partial p^{i, 2}}{\partial x_{2}} & \cdots & \frac{\partial p^{i, 2}}{\partial x_{4 n+2 k}} \\
\vdots & \vdots & & \vdots \\
\frac{\partial p^{i, 2 n+k}}{\partial x_{1}} & \frac{\partial p^{i, 2 n+k}}{\partial x_{2}} & \cdots & \frac{\partial p^{i, 2 n+k}}{\partial x_{4 n+2 k}}
\end{array}\right), \\
B_{i}=\left(\begin{array}{cccc}
\frac{\partial p^{i, 1}}{\partial y_{1}} & \frac{\partial p^{i, 1}}{\partial y_{2}} & \cdots & \frac{\partial p^{i, 1}}{\partial y_{4 n+2 k}} \\
\frac{\partial p^{i, 2}}{\partial y_{1}} & \frac{\partial p^{i, 2}}{\partial y_{2}} & \cdots & \frac{\partial p^{i, 2}}{\partial y_{4 n+2 k}} \\
\vdots & \vdots & & \vdots \\
\frac{\partial p^{i, 2 n+k}}{\partial y_{1}} & \frac{\partial p^{i, 2 n+k}}{\partial y_{2}} & \cdots & \frac{\partial p^{i, 2 n+k}}{\partial y_{4 n+2 k}}
\end{array}\right) .
\end{gathered}
$$

Since $\Phi_{i}^{(0,0)}=N_{i} d f$, where $N_{i}$ is a constant, $i=1,2,3$, then $P((0,0))=0$. So, if we prove that

$$
\operatorname{det}(D)(0,0) \neq 0,
$$

where $D$ is the matrix

$$
\left(\begin{array}{c|c}
A_{1} & B_{1} \\
\hline A_{2} & B_{2} \\
\hline A_{3} & B_{3} \\
\hline A_{4} & B_{4}
\end{array}\right),
$$

then the lemma holds by applying the Implicit Function Theorem to the function $P$ at $(0,0)$.

Assume that the determinant in (7) vanishes. Thus, the columns of the matrix $D$, call them $\left\{v_{1}, \ldots, v_{4(2 n+k)}\right\}$, yield a sistem of $4(2 n+k)$ linearly dependent 
vectors in $\mathbb{R}^{4(2 n+k)}$. Hence, there exist $r_{l}, s_{l} \in \mathbb{R}, l=1, \ldots, 2(2 n+k)$ (with at least one of them nonzero), such that

$$
\sum_{l=1}^{2(2 n+k)}\left(r_{l} v_{l}+s_{l} v_{2(2 n+k)+l}\right)=0,
$$

that is,

$$
\sum_{l=1}^{2(2 n+k)}\left(r_{l} \frac{\partial p^{i, j}}{\partial x_{l}}+s_{l} \frac{\partial p^{i, j}}{\partial y_{l}}\right)(0,0)=0
$$

for any $i \in\{1,2,3,4\}$ and $j \in\{1, \ldots, 2 n+k\}$.

On the other hand,

$$
\begin{gathered}
\frac{\partial p^{i, j}}{\partial x_{2 l-1}}=\operatorname{Real}\left(\int_{\gamma_{j}} \frac{\partial \Phi_{i}^{\Lambda, \Delta}}{\partial \lambda_{l}}\right), \frac{\partial p^{i, j}}{\partial y_{2 l-1}}=\operatorname{Real}\left(\int_{\gamma_{j}} \frac{\partial \Phi_{i}^{\Lambda, \Delta}}{\partial \delta_{l}}\right), \\
\frac{\partial p^{i, j}}{\partial x_{2 l}}=\operatorname{Im}\left(\int_{\gamma_{j}} \frac{\partial \Phi_{i}^{\Lambda, \Delta}}{\partial \lambda_{l}}\right), \frac{\partial p^{i, j}}{\partial y_{2 l}}=\operatorname{Im}\left(\int_{\gamma_{j}} \frac{\partial \Phi_{i}^{\Lambda, \Delta}}{\partial \delta_{l}}\right)
\end{gathered}
$$

for $i \in\{1,2,3\}$ and $l, j \in\{1, \ldots, 2 n+k\}$, and analogously

$$
\begin{gathered}
\frac{\partial p^{4, j}}{\partial x_{2 l-1}}=\operatorname{Im}\left(\int_{\gamma_{j}} \frac{\partial \Phi_{3}^{\Lambda, \Delta}}{\partial \lambda_{l}}\right), \frac{\partial p^{4, j}}{\partial y_{2 l-1}}=\operatorname{Im}\left(\int_{\gamma_{j}} \frac{\partial \Phi_{3}^{\Lambda, \Delta}}{\partial \delta_{l}}\right), \\
\frac{\partial p^{4, j}}{\partial x_{2 l}}=-\operatorname{Real}\left(\int_{\gamma_{j}} \frac{\partial \Phi_{3}^{\Lambda, \Delta}}{\partial \lambda_{l}}\right), \frac{\partial p^{4, j}}{\partial y_{2 l}}=-\operatorname{Real}\left(\int_{\gamma_{j}} \frac{\partial \Phi_{3}^{\Lambda, \Delta}}{\partial \delta_{l}}\right)
\end{gathered}
$$

for $l, j \in\{1, \ldots, 2 n+k\}$.

Thus, if we put $z_{l}=r_{2 l-1}-i r_{2 l}, w_{l}=s_{2 l-1}-i s_{2 l}, l=1, \ldots, 2 n+k$, the formulae in (8) imply

$$
\operatorname{Real}\left(\sum_{l=1}^{2 n+k} z_{l} \int_{\gamma_{j}} \frac{\partial \Phi_{i}^{\Lambda, \Delta}}{\partial \lambda_{l}}(0,0)+w_{l} \int_{\gamma_{j}} \frac{\partial \Phi_{i}^{\Lambda, \Delta}}{\partial \delta_{l}}(0,0)\right)=0
$$

for $i \in\{1,2,3\}$ and $l, j \in\{1, \ldots, 2 n+k\}$, and

$$
\operatorname{Im}\left(\sum_{l=1}^{2 n+k} z_{l} \int_{\gamma_{j}} \frac{\partial \Phi_{3}^{\Lambda, \Delta}}{\partial \lambda_{l}}(0,0)+w_{l} \int_{\gamma_{j}} \frac{\partial \Phi_{3}^{\Lambda, \Delta}}{\partial \delta_{l}}(0,0)\right)=0
$$

for $l, j=1, \ldots, 2 n+k$. But from (5) and for each $l, j \in\{1, \ldots, 2 n+k\}$ we have

$$
\begin{aligned}
& \frac{\partial \int_{\gamma_{j}} \Phi_{1}^{\Lambda, \Delta}}{\partial \lambda_{l}}(0,0)=\left(\frac{h(g-1 / g)}{2}\right)^{\prime}(0) \int_{\gamma_{j}} \xi_{l}, \\
& \frac{\partial \int_{\gamma_{j}} \Phi_{1}^{\Lambda, \Delta}}{\partial \delta_{l}}(0,0)=\left(\frac{h(g-1 / g)}{2}\right)(0) \int_{\gamma_{j}} \xi_{l}, \\
& \frac{\partial \int_{\gamma_{j}} \Phi_{2}^{\Lambda, \Delta}}{\partial \lambda_{l}}(0,0)=i\left(\frac{h(g+1 / g)}{2}\right)^{\prime}(0) \int_{\gamma_{j}} \xi_{l}, \\
& \frac{\partial \int_{\gamma_{j}} \Phi_{2}^{\Lambda, \Delta}}{\partial \delta_{l}}(0,0)=i\left(\frac{h(g+1 / g)}{2}\right)(0) \int_{\gamma_{j}} \xi_{l},
\end{aligned}
$$




$$
\begin{aligned}
& \frac{\partial \int_{\gamma_{j}} \Phi_{3}^{\Lambda, \Delta}}{\partial \lambda_{l}}(0,0)=h^{\prime}(0) \int_{\gamma_{j}} \xi_{l}, \\
& \frac{\partial \int_{\gamma_{j}} \Phi_{3}^{\Lambda, \Delta}}{\partial \delta_{l}}(0,0)=h(0) \int_{\gamma_{j}} \xi_{l},
\end{aligned}
$$

and so (9) and (10) become

$$
\begin{gathered}
\operatorname{Real}\left(\sum_{l=1}^{2 n+k}\left(z_{l}(h(g-1 / g))^{\prime}(0)+w_{l}(h(g-1 / g))(0)\right) \int_{\gamma_{j}} \xi_{l}\right)=0, \\
\operatorname{Im}\left(\sum_{l=1}^{2 n+k}\left(z_{l}(h(g+1 / g))^{\prime}(0)+w_{l}(h(g+1 / g))(0)\right) \int_{\gamma_{j}} \xi_{l}\right)=0, \\
\sum_{l=1}^{2 n+k}\left(z_{l} h^{\prime}(0)+w_{l} h(0)\right) \int_{\gamma_{j}} \xi_{l}=0
\end{gathered}
$$

for any $j \in\{1, \ldots, 2 n+k\}$. From (12), the meromorphic 1-form on $M$ defined by $\sum_{l=1}^{2 n+k}\left(z_{l} h^{\prime}(0)+w_{l} h(0)\right) \xi_{l}$ is exact, and hence by Theorem 1 we obtain

$$
z_{l} h^{\prime}(0)+w_{l} h(0)=0, \quad l=1, \ldots, 2 n+k .
$$

From $(1), h(0) \neq 0$, and so $w_{l}=-z_{l} h^{\prime}(0) / h(0), l=1, \ldots, 2 n+k$. If we replace $w_{l}$ by this expression, the equations (12) become:

$$
\operatorname{Real}\left(\sum_{l=1}^{2 n+k} z_{l} h(0) g^{\prime}(0)\left(1+1 / g^{2}(0)\right) \int_{\gamma_{j}} \xi_{l}\right)=0,
$$

$$
\operatorname{Im}\left(\sum_{l=1}^{2 n+k} z_{l} h(0) g^{\prime}(0)\left(1-1 / g^{2}(0)\right) \int_{\gamma_{j}} \xi_{l}\right)=0
$$

for $j=1, \ldots, 2 n+k$.

Assertion. Without loss of generality, we can assume that $g^{\prime}(0) \neq 0$ and $g(0) \in$ $\mathbb{R}-\{1,-1\}$.

To see this, take a point $z_{0} \in \mathbb{D}$ such that $g^{\prime}\left(z_{0}\right) \neq 0$, and let $L$ be a Möbius transformation leaving $\mathbb{D}$ invariant and such that $L(0)=z_{0}$ (for instance, $L(z)=$ $\left.\left(z+z_{0}\right) /\left(\overline{z_{0}} z+1\right)\right)$. Then replace $x$ by the minimal immersion $x \circ L$. The new Gauss map is $g \circ L$ and $(g \circ L)^{\prime}(0) \neq 0$.

Moreover, after composing with a suitable rigid motion, we can also suppose that $g(0) \in \mathbb{R}-\{1,-1\}$.

Taking into account the former assertion and (13), we get:

$$
\sum_{l=1}^{2 n+k} z_{l} \int_{\gamma_{j}} \xi_{l}=0
$$

for $j=1, \ldots, 2 n+k$. Therefore the 1 -form $\sum_{l=1}^{2 n+k} z_{l} \xi_{l}$ is exact on $M$, and by using Theorem 1 once again we get $z_{l}=0, l=1, \ldots, 2 n+k$. This implies $w_{l}=0$, $l=1, \ldots, 2 n+k$, and so $r_{l}, s_{l}=0, l=1, \ldots, 2(2 n+k)$, which is absurd. 
We can now apply the Implicit Function Theorem and obtain the existence of an open subset $U \subset \mathbb{C}, 0 \in U$, and a differentiable (in fact real analytic) map

$$
\Psi=\left(\Psi_{1}, \Psi_{2}\right): U \longrightarrow \mathbb{C}^{2 n+k} \times \mathbb{C}^{2 n+k}
$$

such that $P\left(\left(\lambda, \Psi_{1}(\lambda)\right), \Psi_{2}(\lambda)\right)=0, \lambda \in U$. This means that the 1 -form $\Phi_{3}^{\left(\lambda, \Psi_{1}(\lambda)\right), \Psi_{2}(\lambda)}$ is exact and $\Phi_{i}^{\left(\lambda, \Psi_{1}(\lambda)\right), \Psi_{2}(\lambda)}, i=1,2$, have no real periods on $C_{A}^{\left(\lambda, \Psi_{1}(\lambda)\right)}$. Since $(\lambda, \Psi(\lambda)) \in \Sigma$, we deduce that $\left|\left(\lambda, \Psi_{1}(\lambda)\right)\right|<\varepsilon, \lambda \in U$.

Note also that when $\lambda \neq 0$, Lemma 2 yields that $C_{A}^{\left(\lambda, \Psi_{1}(\lambda)\right)}$ has $k+1$ holes. In particular, by Remark 3 the three 1 -forms $\Phi_{i}^{\left(\lambda, \Psi_{1}(\lambda)\right), \Psi_{2}(\lambda)}, i=1,2,3$ satisfy (1). In general $\Psi \neq 0$, but this is not significant because these facts remain true even in the case $\Psi=0$ (see Remark 4).

Hence, $x^{\lambda}=x^{\left(\lambda, \Psi_{1}(\lambda)\right), \Psi_{2}(\lambda)}: C_{A}^{\left(\lambda, \Psi_{1}(\lambda)\right)} \longrightarrow \mathbb{R}^{3}, \lambda \in U-\{0\}$, defines a conformal minimal immersion.

This concludes the proof.

In what follows, when $\lambda \in U-\{0\}$, we write, for simplicity, $\Phi_{i}^{\lambda}, i=1,2,3, C^{\lambda}$, $C_{A}^{\lambda}, G^{\lambda}, g^{\lambda}$ and $h^{\lambda}$ instead of $\Phi_{i}^{\left(\lambda, \Psi_{1}(\lambda)\right), \Psi_{2}(\lambda)}, i=1,2,3, C^{\left(\lambda, \Psi_{1}(\lambda)\right)}, C_{A}^{\left(\lambda, \Psi_{1}(\lambda)\right)}$, $G^{\left(\lambda, \Psi_{1}(\lambda)\right)}, g^{\left(\lambda, \Psi_{1}(\lambda)\right)}$ and $h \circ G^{\left(\lambda, \Psi_{1}(\lambda)\right)}$, respectively.

Remark 4. If $\lambda \in U-\{0\}$, then $\Lambda \neq 0$. Thus Lemma 1 and the comments which follow Remark 1 imply that the order of the pole of the meromorphic function $G^{\lambda}$ at $Q_{0}$ is not a multiple of the pole of $f$ at $Q_{0}$. Hence the function $G^{\lambda}$ is not a rational function of $f$, and so $x^{\lambda}$ is not an (unbranched) covering of $x$.

Note that from Lemma $2, C^{\lambda}$ is conformally equivalent to a genus $n$ compact surface minus $k+1$ pairwise disjoint disks with piecewise analytic boundary. In addition, $C_{A}^{\lambda}$ is a surface with (possibly void) piecewise analytic boundary.

The minimal immersions $\left\{x^{\lambda}\right\}_{\lambda \in U-\{0\}}$ preserve some properties of $x$. The most important one is completeness. In the case of nonempty boundary, completeness means that any divergent curve in $C_{A}^{\lambda}$ has infinite length.

Theorem 3. If $x: A \longrightarrow \mathbb{R}^{3}$ is complete then, for any $\lambda \in U-\{0\}$, the immersion $x^{\lambda}: C_{A}^{\lambda} \longrightarrow \mathbb{R}^{3}$ is complete.

Proof. The metric $d s^{2}$ on $C_{A}^{\lambda}$ induced by $x^{\lambda}$ is given by

$$
d s^{2}=\sum_{i=1}^{3}\left|\Phi_{i}^{\lambda}\right|^{2}
$$

We know that $C^{\lambda}$ has $k+1$ holes, and each hole contains in its interior one (and only one) point $Q_{i}, i=0,1, \ldots, k$. This means that

$$
C^{\lambda}=M-\left(\bigcup_{j=0}^{k} \bar{E}_{j}^{\lambda}\right),
$$

where $\bar{E}_{j}^{\lambda}$ is topologically a closed disk contained in $M, Q_{j} \in E_{j}^{\lambda}$ (where $E_{j}^{\lambda}$ is the interior of $\bar{E}_{j}^{\lambda}$ ) and $\bar{E}_{i}^{\lambda} \cap \bar{E}_{j}^{\lambda}=\emptyset, i \neq j$ (in fact $\bar{E}_{j}^{\lambda} \subset D_{j}, j=0,1, \ldots, k$ ). Note also that, by definition, $C_{A}^{\lambda}-C^{\lambda}$ is the union of a set of piecewise analytic curves lying in $\bigcup_{j=0}^{k} \partial E_{j}^{\lambda}$. 
From the definition of $F^{\Delta}, \Delta \in \mathbb{C}^{2 n+k}$, the poles of this function lie in $\left\{Q_{0}, Q_{1}\right.$, $\left.\ldots, Q_{k}\right\}$, and so the same holds for $F^{\Psi_{2}(\lambda)}, \lambda \in U-\{0\}$. Hence we can find two positive constants $k_{1}(\lambda)$ and $k_{2}(\lambda)$ such that

$$
k_{1}(\lambda)<\left|\operatorname{Exp}\left(F^{\Psi_{2}(\lambda)}(Q)\right)\right|<k_{2}(\lambda), \forall Q \in C_{A}^{\lambda} .
$$

Therefore, $d s^{2}$ is complete if and only if the metric $d s_{1}^{2}=\left|\operatorname{Exp}\left(-2 F^{\Psi_{2}(\lambda)}\right)\right| d s^{2}$ is complete. From the definition of $\Phi_{i}^{\lambda}, i=1,2,3$, we have

$$
d s_{1}^{2}=\frac{\left|h^{\lambda}\right|^{2}\left(1+\left|g^{\lambda}\right|^{2}\right)^{2}}{2\left|g^{\lambda}\right|^{2}}|d f|^{2} .
$$

On the other hand, observe that $G^{\lambda}$ is holomorphic on $M_{0}$ and that $d f$ has no zeroes on $M_{0}$. Hence we deduce that the meromorphic function $d f / d G^{\lambda}$ has no zeroes on $M_{0}$, and so by (14) there exists a positive constant $k(\lambda)$ satisfying

$$
k(\lambda)<\frac{|d f|^{2}}{\left|d G^{\lambda}\right|^{2}}(Q), \quad \forall Q \in C_{A}^{\lambda} .
$$

As the immersion $x$ is complete, the metric $d s_{0}^{2}$ on $A$ defined by

$$
d s_{0}^{2}=\frac{|h|^{2}\left(1+|g|^{2}\right)^{2}}{2|g|^{2}}|d z|^{2}
$$

is complete. Therefore, the (finitely branched) metric $\left(G^{\lambda}\right)^{*}\left(d s_{0}^{2}\right)$ on $C_{A}^{\lambda}$ is complete too. Taking (16) and (17) into account, we have

$$
d s_{1}^{2}=\left(G^{\lambda}\right)^{*}\left(d s_{0}^{2}\right) \frac{|d f|^{2}}{\left|d G^{\lambda}\right|^{2}}>k(\lambda)\left(G^{\lambda}\right)^{*}\left(d s_{0}^{2}\right),
$$

and so $d s_{1}^{2}$ is complete. The theorem holds.

As the Gauss map $g^{\lambda}$ of $x^{\lambda}$ is given by $g^{\lambda}=g \circ G^{\lambda}$, where $G^{\lambda}$ is a meromorphic function, $x^{\lambda}$ has any property of $x$ that depends on the Gauss map and is preserved by a branched covering. We emphasize the following ones:

Corollary 1. If $x: \mathbb{D} \longrightarrow \mathbb{R}^{3}$ is complete and nonflat and $g$ omits four points of the sphere, then, for any $\lambda \in U-\{0\}, x^{\lambda}: C^{\lambda} \longrightarrow \mathbb{R}^{3}$ is complete and nonflat and $g^{\lambda}$ omits four points too.

Proof. For any $\lambda \in U-\{0\}$, the Gauss maps $g^{\lambda}$ and $g$ of $x^{\lambda}$ and $x$, respectively, have the same image. Moreover, by Theorem 3 the completeness is preserved.

Complete nonflat minimal surfaces whose Gauss map omits four points of the sphere are critical from the point of view of Fujimoto's theorem [FU1, FU2]. The classical example is Sherk's doubly periodic minimal surface. We can apply the above corollary to the universal covering of this surface and so construct complete examples with arbitrary topology whose Gauss map also omits four points.

On the other hand, a large family of surfaces of this type was exhibited by K. Voss $[\mathrm{V}]$ (see also $[\mathrm{O}]$ ). Voss examples are simply connected, and Corollary 1 works for them too.

Corollary 2. Suppose that $x: \mathbb{D} \longrightarrow \mathbb{R}^{3}$ is complete and nonflat. Assume also that the third 1-form $\Phi_{3}$ of the Weierstrass representation of $x$ is bounded, that is, $\Phi_{3}(z)=h(z) d z$ and $|h(z)|<K, \forall z \in \mathbb{D}$.

Then, for any $\lambda \in U-\{0\}$, the minimal immersion $x^{\lambda}: \mathbb{D} \longrightarrow \mathbb{R}^{3}$ is complete and its third coordinate function is bounded. 
Proof. Without loss of generality, we can suppose that the homology basis $\left\{\gamma_{1}, \ldots\right.$, $\left.\gamma_{2 n}\right\}$ of $M$ satisfies

$$
\bigcup_{j=1}^{2 n} \gamma_{j} \subset M-\bigcup_{i=0}^{k} \bar{D}_{i}
$$

and that

$$
\Omega=M-\left(\bigcup_{j=1}^{2 n} \gamma_{j}\right)
$$

is simply-connected.

Take $w$ a conformal parameter on $\Omega$, and observe that (15) and our hypothesis imply that the function $\Phi_{3}^{\lambda}(w) / d w$ is bounded on $C^{\lambda}-\left(\bigcup_{j=1}^{2 n} \gamma_{j}\right)$, and so the same holds for the function $x_{3}^{\lambda}$. As the set $\bigcup_{j=1}^{2 n} \gamma_{j}$ is compact, $x_{3}^{\lambda}$ is in fact bounded on $C^{\lambda}$.

For the completeness, use Theorem 3.

By different methods, L.P. Jorge and F. Xavier [JX1] and later F. F. Brito [B1] discovered examples of complete nonflat minimal immersions $x: \mathbb{D} \longrightarrow \mathbb{R}^{3}$ with $\Phi_{3}(z)=h(z) d z, h$ bounded. Hence, Corollary 2 yields complete nonflat examples of high topology with the third coordinate function bounded.

Corollary 3. If $x: A \longrightarrow \mathbb{R}^{3}$ is nonflat and stable, then for any $\lambda \in U-\{0\}$, the immersion $x^{\lambda}: C_{A}^{\lambda} \longrightarrow \mathbb{R}^{3}$ is also nonflat and stable.

Furthermore, if $\partial A \neq \emptyset$ and $x$ is complete, the same holds for $x^{\lambda}$.

Proof. The index of a minimal surface can be computed in terms of its Gauss map (see $[\mathrm{FC}]$ ). In fact, the index of $x$ is the index of the quadratic form associated to the Jacobi operator $\Delta+|\nabla g|$ on $A$. Recall that stability means index zero.

If this operator has index zero, then the same holds for the corresponding operator associated to the meromorphic function $g \circ G$, where $G$ is any (branched) covering of $A$ (see, for instance, [FCS]).

By Theorem 3, the completeness is preserved. This proves the corollary.

A way to construct simply-connected stable minimal surfaces consists of taking a piece of a minimal surface whose spherical image has area less than $2 \pi$ (see $[\mathrm{BC}]$ ).

Particularly interesting is the case of the helicoid. Let $A$ be one of the two pieces of the helicoid, both bounded by the same straight line, whose Gauss map covers a closed hemisphere $H_{a}=\left\{u \in \mathbb{S}^{2}:\langle u, a\rangle \geq 0\right\}, a \in \mathbb{S}^{2}$. It is clear that conformally $A=\overline{\mathbb{D}}-\left\{P_{0}\right\}$, where $P_{0} \in \partial \mathbb{D}$, and $x: A \longrightarrow \mathbb{R}^{3}$ is complete with boundary. Furthermore, this surface is stable because the function $f=\langle g, a\rangle$ is positive and satisfies $\Delta f+|\nabla g| f=0$ (see [FCS]). Then, the surfaces arising from $x$ in Corollary 3 are orientable, stable, complete with boundary and of arbitrary topology.

It is interesting to note that one can deform (when $\lambda$ tends to zero) any surface $x^{\lambda}$ in the above three large families, by surfaces of the same kind, into a branched covering of a plane. The topology and the number of ends are preserved in this deformation.

Finally, we mention the following interesting consequence of Lemma 3:

Corollary 4. There exist orientable nonflat minimal surfaces with three ends and arbitrary genus $n>0$ in $\mathbb{R}^{3}$ of the following kinds: complete surfaces whose Gauss 
map omits three points of the sphere, complete surfaces with a bounded coordinate function, and stable surfaces which are incomplete or complete with boundary.

\section{REFERENCES}

[BC] J.L. Barbosa and M.P. Do Carmo. On the size of a stable minimal surface in $\mathbb{R}^{3}$. Amer. J. Math., 98 (1976), 515-528. MR 54:1292

[B1] F. F. De Brito. Power series with Hadamard gaps and hyperbolic complete minimal surfaces. Duke Math. J., 68 (1992), N. 2, 297-300. MR 94d:53012

[B2] F.F. De Brito. Many-ended complete minimal surfaces between two parallel planes. Preprint.

$[\mathrm{CH}]$ S. Y. Cheng, Eigenfunctions and nodal sets, Comm. Math. Helv. 51 (1976) 43-55. MR 55: 1661

[CS] C. Costa and P.A.Q. Simoes. Complete minimal surfaces of arbitrary genus in a slab of $\mathbb{R}^{3}$. Ann. Inst. Fourier (Grenoble) 46 (1996), 535-546. MR 97e:53015

[F-K] H. M. Farkas and I. Kra. Riemann surfaces. Grad. Texts in Math., 72, Springer Verlag, Berlin, 1980. MR 82c:30067

[FC] D. Fischer-Colbrie. On complete minimal surfaces with finite Morse index in threemanifolds. Invent. Math., 82 (1985), 121-132. MR 87b:53090

[FCS] D. Fischer-Colbrie and R. Schoen. The structure of complete stable minimal surfaces in 3-manifolds of non negative scalar curvature. Comm. of Pure and Appl. Math., 33 (1980), 199-211. MR 81i:53044

[FU1] H. Fujimoto. On the number of exceptional values of the Gauss maps of minimal surfaces. J. Math. Soc. Japan, 40 (1988), 235-247. MR 89b:53013

[FU2] H. Fujimoto. Modified defect relations for the Gauss map of minimal surfaces. J. of Diff. Geom., 29 (1989), 245-262. MR 89m:53012

[JX1] L.P.M. Jorge and F. Xavier. A complete minimal surface in $\mathbb{R}^{3}$ between two parallel planes. Ann. of Math., 112 (1980), 203-206. MR 82e:53087

[L] F.J. Lopez. A nonorientable complete minimal surface in $\mathbb{R}^{3}$ between two parallel planes. Proc. Amer. Math. Soc., 103 (1988), 913-917. MR 89f:53009

[O] R. Osserman. A survey of minimal surfaces. Dover Publications, New York, second edition, 1986. MR 87j:53012

[RT] H. Rosenberg and E. Toubiana. A cylindrical type complete minimal surface in a slab of $\mathbb{R}^{3}$. Bull. Sci. Math. 111 (1987), 241-245. MR 88k:53019

[V] K. Voss. Über vollständige minimalflächen. L'Enseigment Math., 10 (1964), 316-317.

[X] F. Xavier. The Gauss map of a complete nonflat minimal surface cannot omit 7 points of the sphere. Ann. of Math., 113 (1981), 211-214; 115 (1982), 667. MR 82b:53015; MR 83h:53016

Departamento de Geometría y Topología, Facultad de Ciencias, Universidad de Granada, 18071 - Granada (SPAin)

E-mail address: fjlopez@goliat.ugr.es 